\title{
Morphological Analysis of Polymers on Hair Fibers by SEM and AFM
}

\author{
Valéria Fernandes Monteiro*, Aline Martins Duboc Natal, \\ Luís Edmundo Bastos Soledade, Elson Longo \\ CMDMC, LIEC, UFSCAr \\ Rod. Washington Luís, km 235, C.P. 676, 13565-905 São Carlos - SP, Brazil
}

Received: February 4, 2003; Revised: July 27, 2003

\begin{abstract}
The polyquaternium $7{ }^{\circledR}$ polymer is widely used in cosmetic formulations. Morphologic alterations in hair fibers were observed after the application of the polyquaternium $7{ }^{\circledR}$ polymer, using SEM and AFM. Continuous applications of this product indicated that it accumulates on the fibers, improving the aspect of the hair surface. Quantitative analysis of the images obtained by AFM was undertaken. The data obtained for the hair surface roughness indicates that the fibers treated with the polymer presented higher roughness than the untreated hair fibers.
\end{abstract}

Keywords: Polymers, SEM, AFM

\section{Introduction}

The interactions between apolar solids and solids presenting low polarity take place by van der Waals type forces. These forces are labile and difficult to be analyzed by conventional methods. The hair is constituted basically by the keratin biopolymer. The keratin is formed by amino acids resulting in complex polymeric structures of low polarity. These polymeric structures interact with other polymers causing changes in the surfaces ${ }^{1}$.

The study of the behavior of human hair surface is of great interest for the cosmetic science. The hair surface is responsible for the diffusion of compounds that promote changes in the cuticular surface, such as the cosmetic products that are deposited on the surface ${ }^{1}$.

Polymers are usually used in hair cosmetic formulations for the improvement of the hair surface. The polymeric materials adhere to the hair surface by van der Waals type forces. Several techniques have been proposed for the evaluation of the morphologic changes in the hair surface after treatments with solutions containing polymers ${ }^{1,3}$.

Cationic polymers are very used in shampoo formulations. These compounds added to the formulation lead to several surface modifications that range from the formation of a protecting film on the hair fibers to the synergic action that improves the performance of other products toward the improvement of the features related to the aspect of hair, such as softness and easiness of combing. These structures

*e-mail: valeria@liec.ufscar.br

Trabalho apresentado no XV CBECIMAT, Natal - RN, Novembro de 2002. interact with other polymeric structures, resulting in changes in the surface ${ }^{1}$.

The polyquaternium $7 \circledR$ polymer is thoroughly used in cosmetic formulations. It is a cationic copolymer constituted by acrylamide and diallyldimethyl ammonium chloride.

Morphological changes of hair fibers, such as the ones due to the adsorption of polymers on hair fibers can be observed by means of SEM and AFM ${ }^{1,3}$.

The analysis based on SEM allows to evaluate gradual changes in the surface structure of human hair. Ruetsch et al., using field emission scanning electron microscopy (FESEM), monitored the effects of UV radiation on the physical nature of hair fibers. They observed solubilization of melanin granules that causes the loss of color of hair ${ }^{2}$.

Atomic force microscopy, (AFM), has extensively proven to be useful for imaging nonconductive substrates. In this method, a sharp tip attached to a cantilever is used to measure the surface topography of the sample, which is scanned in the xy plane. The displacement of the tip, detected by a reflected laser beam and photodiode is used to adjust the sample position in the $\mathrm{z}$ direction in order to maintain a constant force. The feedback voltage provides the topographical ( $\mathrm{z}$ ) data. The attractive or repulsive forces that arise from the interactions between the surface of the sample and tip generate the digital images. The forces involved 
are of the order of $10^{-9} \mathrm{~N}$. AFM has two modes of applied force, contacting and noncontacting modes. In the contacting mode, the force that the cantilever exerts on the sample caused by displacements in the z-axis of the piezoelectric sensor, allows the quantification of surface properties of the material being analyzed. In noncontacting mode the image is obtained by tapping the surface with an oscillating tip that measures the van der Waals interactions ${ }^{3,4}$.

Mattoso et al. ${ }^{4}$ compared the AFM and SEM techniques to be applied in the analysis of polymeric materials. They indicate the advantages of AFM in terms of easier imaging of soft samples and obtaining better resolution of images at molecular and/or atomic scales.

Swift and Smith ${ }^{5}$, using AFM, had verified that the cuticular surfaces of hair did not appear to be as smooth as had been previously supposed to be, when evaluated by SEM. They had observed that different features of the hair fibers gradually change along its length from the root to the end of the fiber.

This paper presents results of the use of SEM and AFM to investigate the changes in the morphology of hair fibers submitted and not to a solution containing polyquaternium $7 \AA$ polymer. The roughness of the surface is a feature of the hair morphology that will be herein discussed.

\section{Experimental}

\subsection{Material}

Untreated black Caucasian hair was obtained from De Meo Brothers, New York, USA. Hair tresses weighing approximately $1.0 \mathrm{~g}$ each one were prepared for this work.

\subsection{Testing}

\section{Bleaching:}

The bleach solution was prepared by mixing a $6 \%$ hydrogen peroxide $\left(\mathrm{H}_{2} \mathrm{O}_{2}\right)$ solution with a concentrated ammonium hydroxide solution and ammonium persulfate powder in a 2:1:1 weight proportion, respectively. The hair tresses remained in the bleach solution for $30 \mathrm{~min}$. The bleaching of the hair tresses without any further treatment is hereinafter called control bleached treatment.

Hydrogen peroxide is normally used in hair paints. Hairs damaged by the action of oxidizing agents such as peroxides probably adsorb a higher amount of polymers, due to the higher irregularity of the damaged hair surfaces, what increases the contact area and the number of the fiber surface charges ${ }^{1}$.

\section{Treatment with polyquaternium $7 ®$ polymer:}

The polyquaternium $7 \AA$ polymer, see structure in Fig. 1, was acquired from Ciba Company.

The hair tresses were washed 5 times with a solution containing $1 \%$ of polyquaternium $7 \circledR .200 \mu \mathrm{l}$ of the solu-

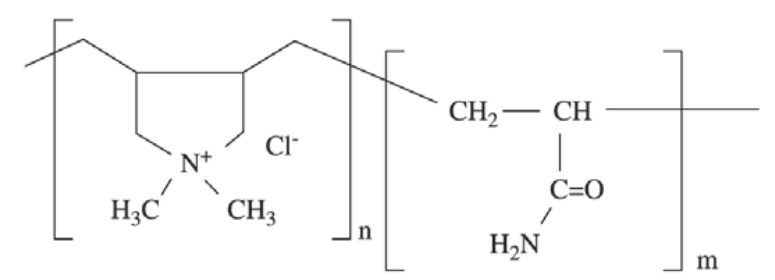

Figure 1. Polyquaternium $7 \circledR$ structure.

tion were kept for $30 \mathrm{~s}$ in contact with the hair fibers, which were rinsed in distilled water for $1 \mathrm{~min}$ and then dried at room temperature. This procedure represents one washing. The treatment with the polymer is always performed in hair tresses previously submitted to bleaching.

Two techniques were used to evaluate the hair surface polymer interaction, as well as to investigate the hair bleaching process:

The Scanning Electron Microscopy (SEM) technique has been used to identify morphological changes on the hair surface.

Longitudinal segments of bleached untreated fibers and fibers treated with the polymer were mounted in an aluminum substrate at an angle of $45^{\circ}$ and coated with gold with thickness of approximately $90 \AA$. The hair fiber topography was examined with a ZEISS 940-A digital SEM. The objective was to analyze the morphological alterations in untreated hair and in the polymer-treated hair.

The Atomic Force Microscopy (AFM) technique was used to analyze the hair morphology at different environmental conditions. It allows the direct study of the cuticle roughness. The $1 \%$ polymer solution was analyzed by the deposition of a fine layer on a glass substrate by using a spin-coater.

Atomic force microscopy studies were performed using a Digital III- Instrument under atmospheric conditions at $25^{\circ} \mathrm{C}$. Imaging was obtained in the contact mode using silicon nitride profile tips mounted in a cantilever of $3.6 \mathrm{nN}$ force constant. AFM images were processed using the Nanoscope software.

\section{Results and Discussion}

\subsection{SEM}

Images obtained by SEM of control bleaching treatment showed damaged cuticle edges (Fig. $2 a$ and $2 b$ ). The bleached, hair treated 5 times with polyquaternium $7 \circledR$ solution showed a higher roughness, caused by the polymer deposition on the hair surface (Fig. 3a and 3b).

\section{2. $A F M$}

AFM three-dimensional images present details of the 


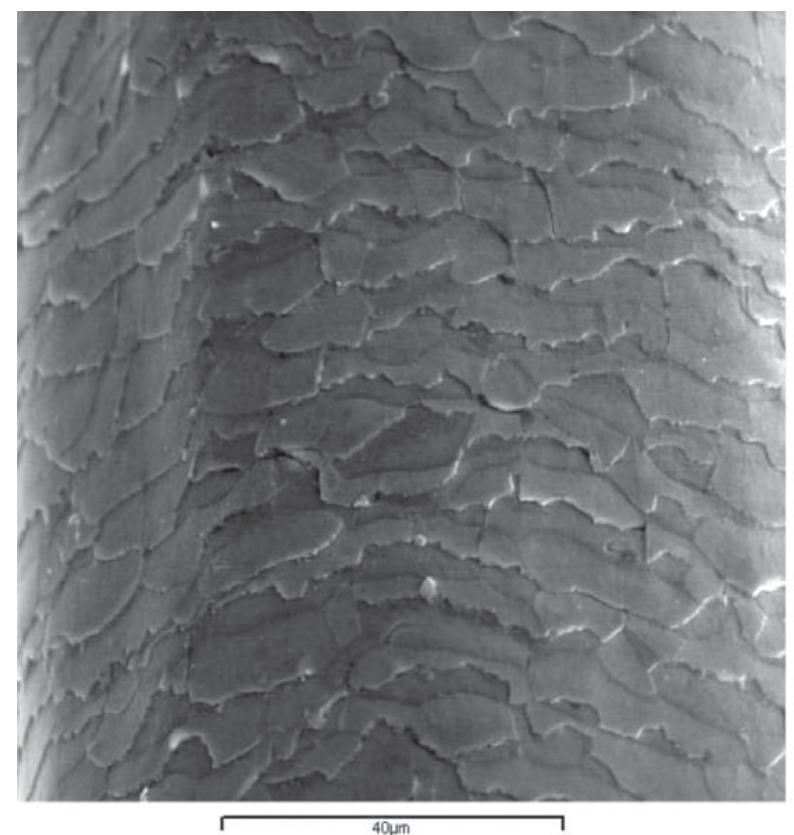

a)

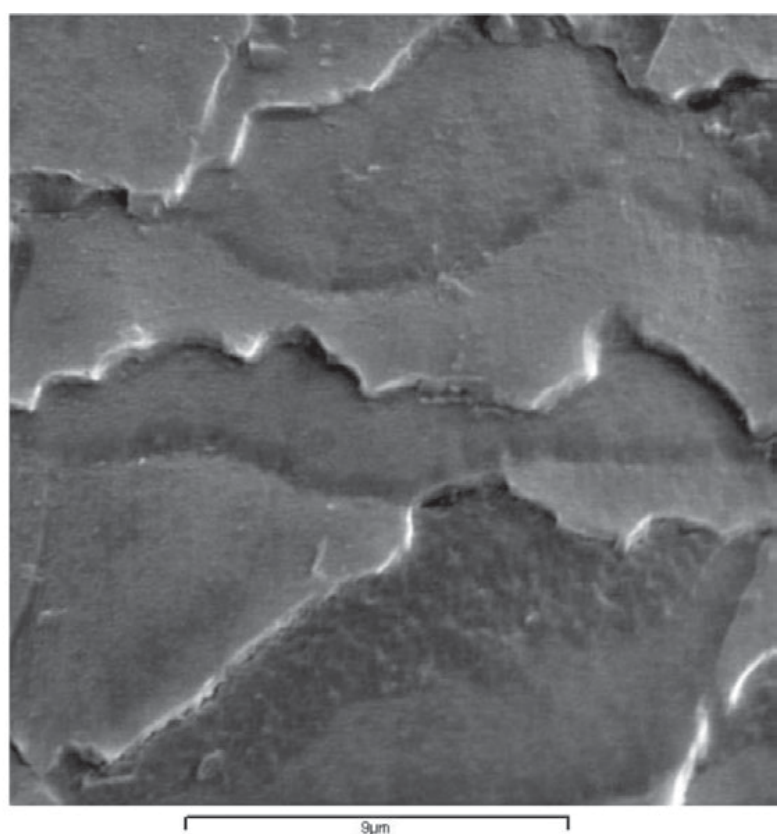

b)

Figure 2. Images obtained by SEM of control bleached hair. a) 1000x; b) 5000×.

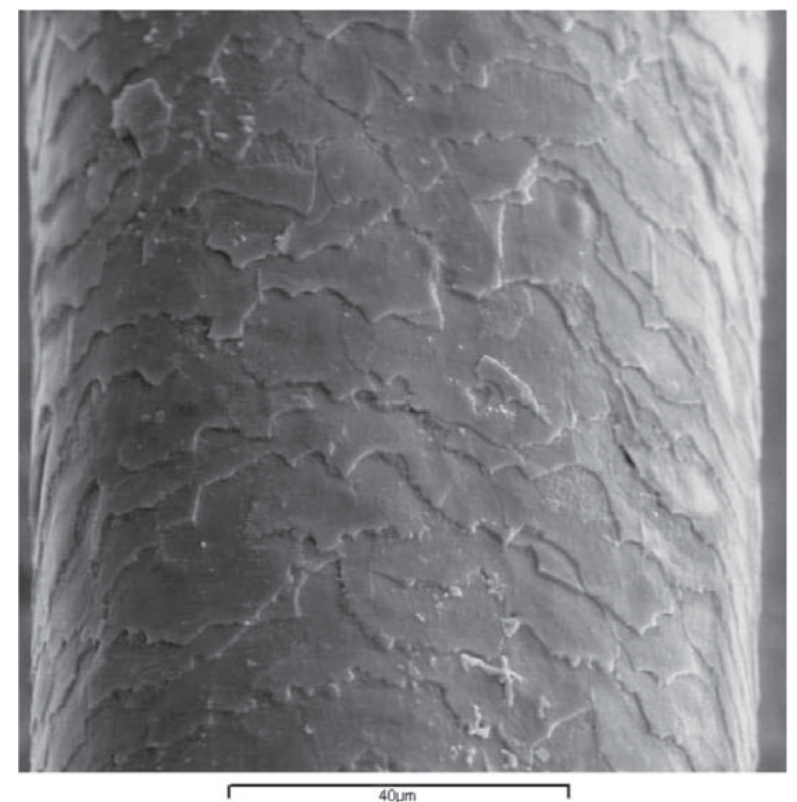

a)

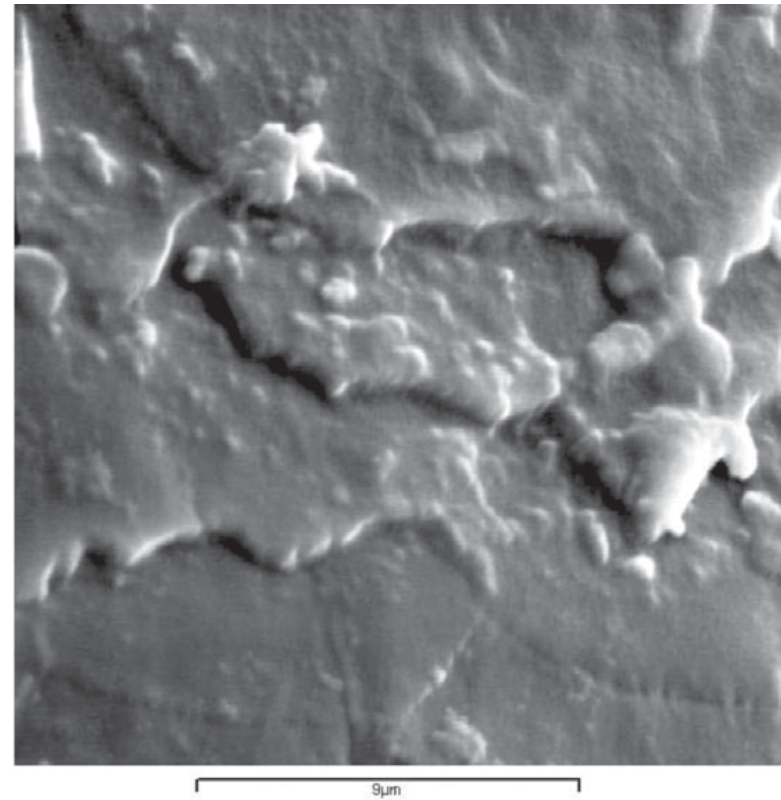

b)

Figure 3. Images obtained by SEM of bleached hair treated with a polyquaternium $7 \circledR$ solution. a) $1000 \times$; b) $5000 \times$.

hair surface of $20 \mu \mathrm{m}$ and $5 \mu \mathrm{m}$ representing an enlargement of $8500 \times$ and $13000 \times$, respectively (Fig. $4 a-4 b, 5 a-5 b$ and $6 a-6 b)$.
In Fig. $4 \mathrm{a}$ and $4 \mathrm{~b}$ are showed the images of the polymer deposited on glass substrate by a spin-coater. The polymer morphology exhibits a rough surface with polymeric parti- 


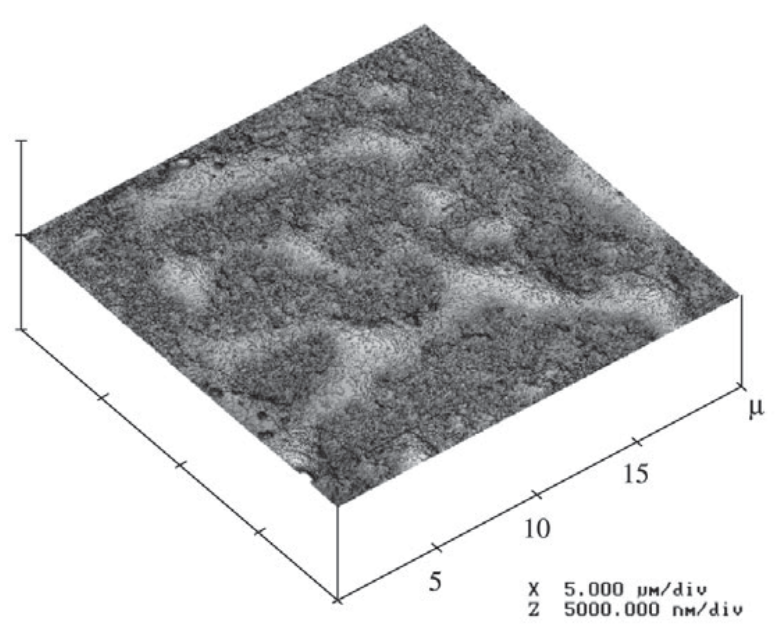

a)

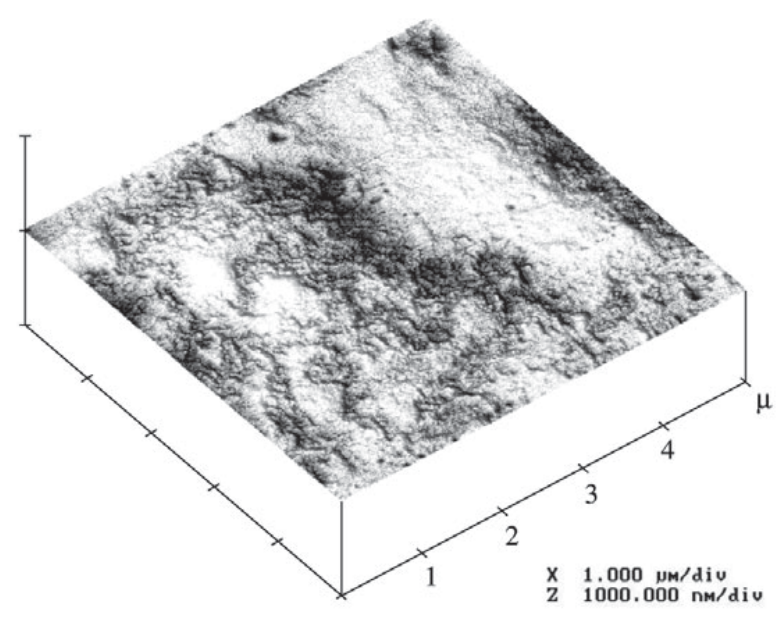

b)

Figure 4. AFM images of polyquaternium 7®polymer deposited on glass substrate: a) $20 \mu \mathrm{m}$; b) $5 \mu \mathrm{m}$.

Table 1. Roughness values (RMS) obtained in $1.719 \times 1.719 \mu \mathrm{m}$ areas, from $20 \times 20 \mu \mathrm{m}$ fields.

\begin{tabular}{cc}
\hline Process & Roughness (nm) \\
\hline $\begin{array}{c}\text { Glass substrate on which } \\
\text { Polyquaternium 7® was deposited. }\end{array}$ & $2.54 \pm 0.16$ \\
$\begin{array}{c}\text { Hair submitted to the } \\
\text { bleaching treatment }\end{array}$ & $17.0 \pm 6.3$ \\
Hair sequentially submitted to \\
$\quad$ bleaching and
\end{tabular}

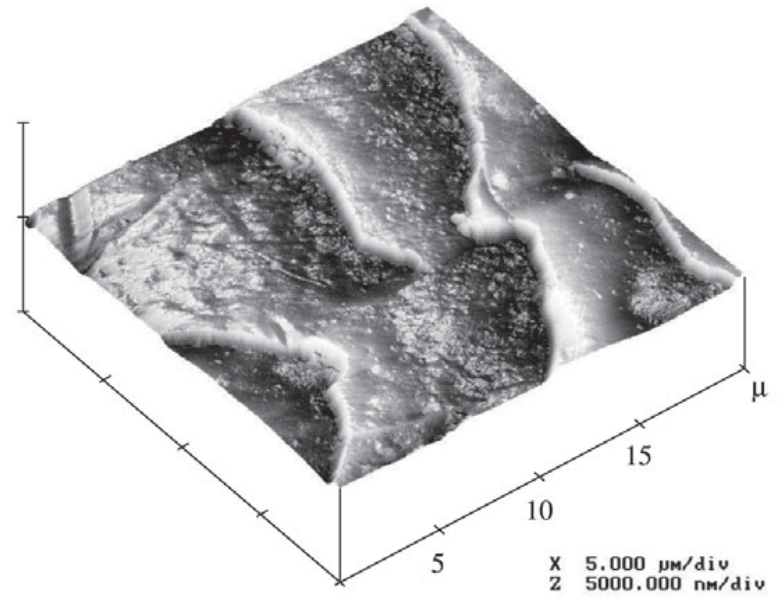

a)

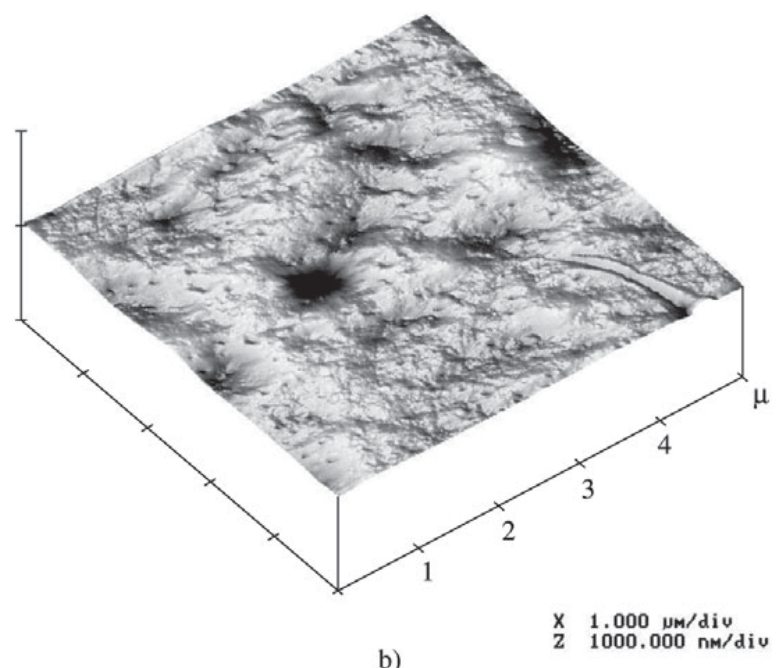

Figure 5. AFM images of control bleached hair: a) $20 \mu \mathrm{m}$; b) $5 \mu \mathrm{m}$.

cles of approximated $2.40 \mu \mathrm{m}$ and their aggregates dispersed on the surface. The value of roughness of this surface was $2.543 \mathrm{~nm} \pm 0.16$, as measured by AFM, see Table 1 .

In Fig. $5 \mathrm{a}-5 \mathrm{~b}$ and $6 \mathrm{a}-6 \mathrm{~b}$ are presented surface images of untreated and polyquaternium $7 \AA$ treated hair fibers, respectively. Figures $5 \mathrm{a}$ and $5 \mathrm{bB}$ show the degraded cuticle surface and the detail of a cuticle face with the formation of pores, caused by the bleaching solution (Fig. 5b).

The root mean square (RMS) surface roughness of the bleached hair can be calculated by using the software of the AFM instrument. These data supply the quantification of the degradations suffered by the hair in the bleaching treat- 


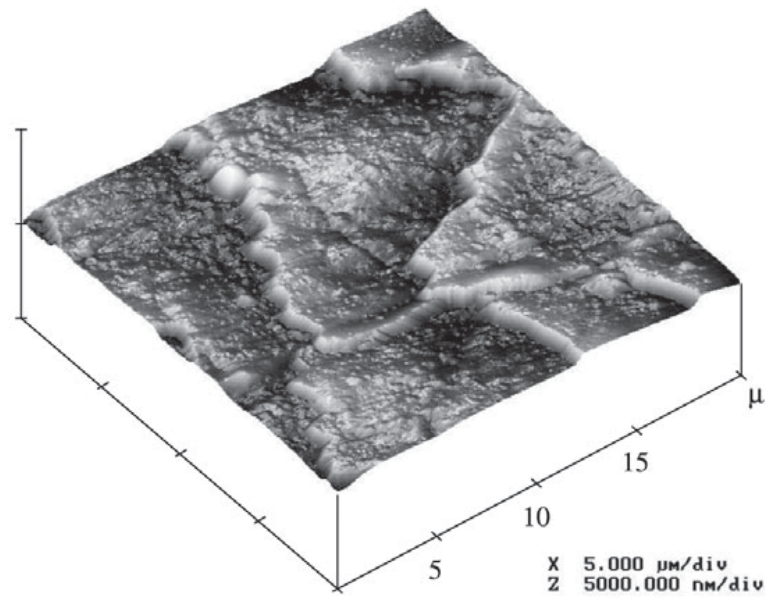

a)

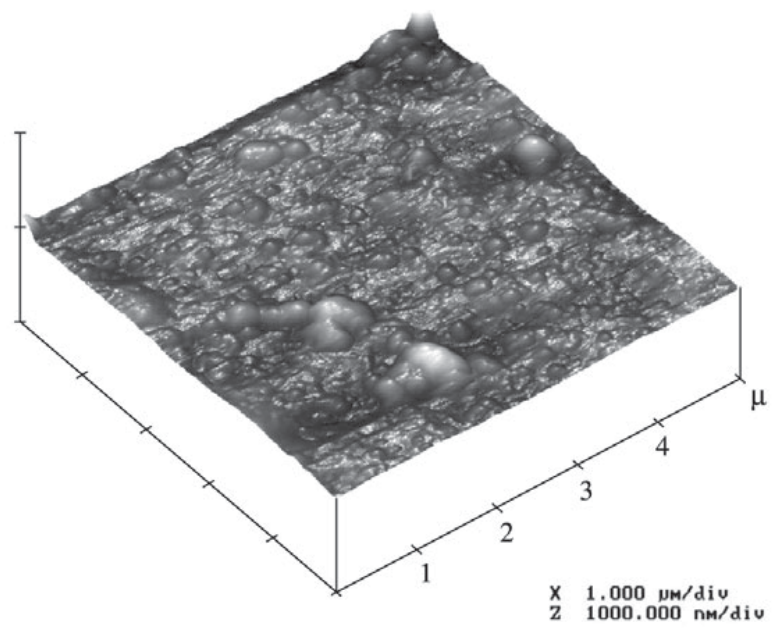

b)

Figure 6. AFM images of bleached hair treated with polyquaternium $7 \AA$ polymer: a) $20 \mu \mathrm{m}$; b) $5 \mu \mathrm{m}$.

ment (Fig. 6a and 6b). The bleached hair, treated with polyquaternium $7 \AA$ presents a higher surface roughness than the control bleached hair images, indicating that the polymer interacted with the hair surface, remaining on the surface, even after the rinses (Fig. 6a - 6b). This result suggests that a kind of film is deposited on the bleached hair. This statement is supported by the diminishment of the static load observed in the hair treated with polyquaternium $7{ }^{\circledR}$. The easier combing observed for the polymer-treated hair supports this reasoning.

The changes in the surface of the hair fibers can be quantified by a software called Nanoscope ${ }^{\circledR}$. In the AFM images

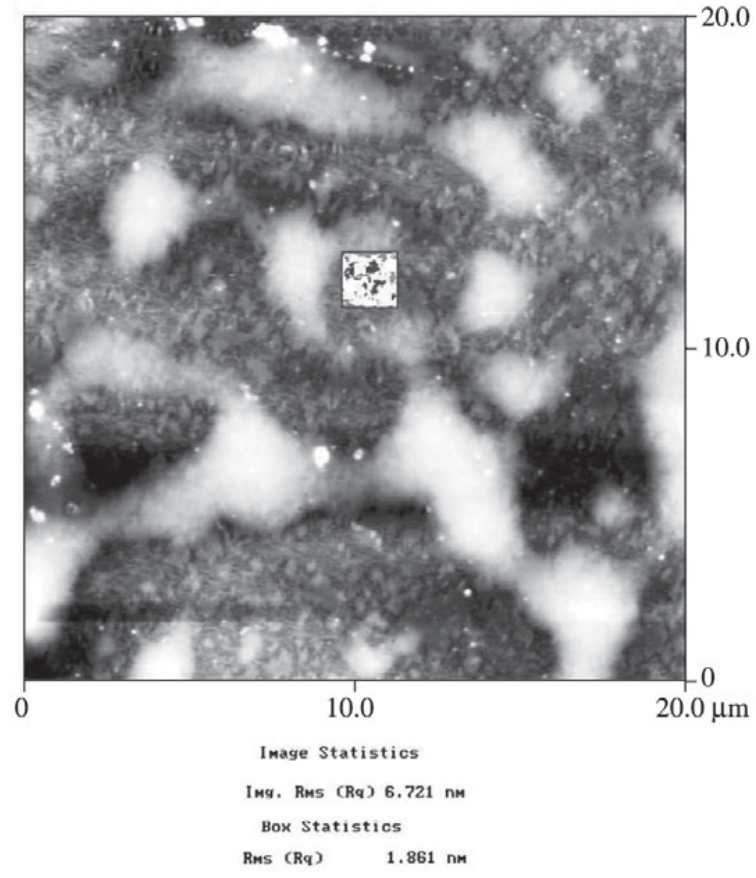

Figure 7. Example of a quantitative roughness analysis of the complete image of polyquaternium7® polymer of a selected region of the image, using the Nanoscope ${ }^{\circledR}$ software.

it is possible to quantify the roughness of the total area and of small selected areas on the cuticular surface (Fig. 7).

The root mean square (RMS) roughness of a bleached hair and of a hair treated with a polyquaternium $7 \AA$ was calculated using the Nanoscope ${ }^{\circledR}$ software ${ }^{\circledR}$. The medium roughness values obtained after each treatment are presented in Table 1, as well the roughness of a surface of the polymer deposited on a glass substrate, as previously commented. Its was observed that the polyquaternium $7 \AA$ polymer deposited on the hair surface increases the hair surface roughness. The change in the bleached hair surface roughness observed upon the treating with the polymer indicates its adsorption on the hair fibers. The interaction between the hair surface and the polymer suggested by the roughness modification can cause a decrease in the static load of the hair, increasing the softness of the hair by the decrease of the attrition among the fibers.

\section{Conclusions}

The use of SEM allowed to evaluate the morphological changes occurred in the bleached hair surface submitted or not to a treatment with polyquaternium $7 \AA$.

This study has shown that AFM is an excellent analytical technique to supply quantitative information on the mor- 
phological changes in the hair under ambient conditions.

The polyquaternium $7 \AA$ polymer interacted with the hair surface, increasing the hair surface roughness. This result, the SEM images, the decrease of static loads and the easier combing of the polymer-treated hair indicate that a kind of film is deposited on the bleached hair, upon such a treatment.

\section{Acknowledgments}

The authors acknowledge the following Brazilian funding support agencies: CNPq, CEPID, CAPES and FAPESP.

\section{References}

1. Robbins, C.R. Chemical and Physical Behavior of Human Hair, SPRINGER - Ed. Verlag, $3^{\text {rd }}$ ed, p. 391,
1994.

2. Ruetsch, S.B.; Kamath, Y.; Weigmann, H.D. Photodegradation of Human Hair: An SEM Study Journal Cosmetology Science, v. 51, p. 103-125, 2000.

3. Goddard, E.D.; Schmitt, R.L. Atomic force microscopy investigation into adsorption of cationic polymers, Cosmetic \& Toiletries, v. 109, p. 55-61, 1994.

4. Mattoso, L.H.C.; Ferreira, F.C.; Herrmann, P.S.P.; Martin, A. R.; Pereira-Da-Silva, M.A.; Paterno, L.G.; Piza, M.A.; Curvelo, A.A.S.; Colnago, L.A.; Denes, F. Atomic Force Microscopy as a Tool for the Evaluation of Natural Fibers and Polymers Surface. The Fifth International Conference on Woodfiber-Plastic Composites, Madison, Wisconsin, p. 43-56, 1999.

5. Swift, J.A.; Smith, J.R. Atomic Force Microscopy of Human Hair, Scanning, v. 22, p. 310-318, 2000. 